\title{
A direct computation of a certain family of integrals
}

\author{
Lorenzo Fornari, Enrico Laeng and Vittorino Pata \\ Department of Mathematics, Politecnico di Milano, Milan, Italy
}

\begin{abstract}
Purpose - The authors propose a rather elementary method to compute a family of integrals on the half line, involving positive powers of $\sin x$ and negative powers of $x$, depending on the integer parameters $n \geq q \geq 1$. Design/methodology/approach - Combinatorics, sine and cosine integral functions.

Findings - The authors prove an explicit formula to evaluate sinc-type integrals.

Originality/value - The proof is not present in the current literature, and it could be of interest for a large audience.
\end{abstract}

Keywords Integral, Sinc function, SinIntegral and CosIntegral functions

Paper type Research paper

In this note, let $n \geq q \geq 1$ be any two given integers. The symbol $\lfloor$.$\rfloor will stand, as usual, for the$ integer part. We consider the family of integrals

$$
I_{n, q}=\int_{0}^{\infty} \frac{(\sin x)^{n}}{x^{q}} d x .
$$

Theorem 1. The following formulae hold

(i) If $n+q$ is even, then

$$
I_{n, q}=\frac{(-1)^{\frac{q-n}{2}} \pi}{2^{n}(q-1) !} \sum_{k=0}^{\left\lfloor\frac{n-1}{2}\right\rfloor}(-1)^{k}\left(\begin{array}{l}
n \\
k
\end{array}\right)(n-2 k)^{q-1} .
$$

(ii) If $n+q$ is odd and $q \geq 2$, then

$$
I_{n, q}=\frac{(-1)^{\frac{q-n+1}{2}}}{2^{n-1}(q-1) !} \sum_{k=0}^{\left\lfloor\frac{n-1}{2}\right\rfloor}(-1)^{k}\left(\begin{array}{l}
n \\
k
\end{array}\right)(n-2 k)^{q-1} \log (n-2 k) .
$$

The formulae above are recorded in the Wolfram MathWorld web page titled Sinc Function [1], which refers to the result as "amazing" and "spectacular". However, the web page omits the proof, citing a 20-year-old online paper that seems not to be available any longer. Nor the proof is reported anywhere else, to the best of our knowledge. Nonetheless, particular instances of $I_{n, q}$ are discussed in several textbooks, typically by means of complex analysis tools (see, e.g. Ref. [2]).

(C) Lorenzo Fornari, Enrico Laeng and Vittorino Pata. Published in Arab Journal of Mathematical Sciences. Published by Emerald Publishing Limited. This article is published under the Creative Commons Attribution (CC BY 4.0) licence. Anyone may reproduce, distribute, translate and create derivative works of this article (for both commercial and non-commercial purposes), subject to full attribution to the original publication and authors. The full terms of this licence may be seen at http:// creativecommons.org/licences/by/4.0/legalcode

Received 16 January 2021 Revised 21 January 2021 Accepted 21 January 2021 
AJMS

27,2

The remaining of the paper is devoted to our proof of Theorem 1 . To this end, for $m \geq 0$, let

$$
P_{m}(x)=\sum_{k=0}^{m} \frac{x^{k}}{k !}
$$

denote the Maclaurin polynomial of $e^{x}$ of order $m$. We agree to set $P_{-1}=0$. Let $Q(x)$ be the Maclaurin polynomial of $(\sin x)^{n}$ of order $q-2$, with $Q=0$ if $q=1$. Since $(\sin x)^{n}$ has a zero of order $n$ at $x=0$, it follows that $Q(x) \equiv 0$ for all $n \geq q \geq 1$. On the other hand, as

$$
(\sin x)^{n}=\frac{1}{(2 i)^{n}} \sum_{k=0}^{n}(-1)^{k}\left(\begin{array}{l}
n \\
k
\end{array}\right) e^{i(n-2 k) x},
$$

we immediately conclude that

$$
Q(x)=\frac{1}{(2 i)^{n}} \sum_{k=0}^{n}(-1)^{k}\left(\begin{array}{l}
n \\
k
\end{array}\right) P_{q-2}(i(n-2 k) x)=0 .
$$

Subtracting the two sums, we obtain

$$
\begin{aligned}
I_{n, q} & =\frac{1}{(2 i)^{n}} \sum_{k=0}^{\left\lfloor\frac{n-1}{2}\right\rfloor}(-1)^{k}\left(\begin{array}{l}
n \\
k
\end{array}\right) \int_{0}^{\infty} \frac{e^{i(n-2 k) x}-P_{q-2}(i(n-2 k) x)}{x^{q}} d x \\
& +\frac{(-1)^{n}}{(2 i)^{n}} \sum_{k=0}^{\left\lfloor\frac{n-1}{2}\right\rfloor}(-1)^{k}\left(\begin{array}{l}
n \\
k
\end{array}\right) \int_{0}^{\infty} \frac{e^{-i(n-2 k) x}-P_{q-2}(-i(n-2 k) x)}{x^{q}} d x .
\end{aligned}
$$

Remark 2. From (1), we also deduce that the equality

$$
\sum_{k=0}^{\left\lfloor\frac{n-1}{2}\right\rfloor}(-1)^{k}\left(\begin{array}{l}
n \\
k
\end{array}\right)(n-2 k)^{q-1}=0
$$

holds for every $n>q \geq 2$, whenever $n+q$ is odd.

We now start from formula (2) but considering the integral on $(\varepsilon, \infty)$ and only at the end we will take the limit $\varepsilon \rightarrow 0$. This allows us to move the integral inside the sum. In what follows $\omega(\varepsilon)$ will denote a generic function of $\varepsilon$, vanishing at 0 as $\varepsilon \rightarrow 0$. Moreover, for $\alpha \neq 0$, let us define

$$
\mathrm{E}_{\varepsilon}(\alpha)=\int_{\varepsilon}^{\infty} \frac{e^{i \alpha x}}{x} d x
$$

Lemma 3. For every $q \geq 1$, every $\varepsilon>0$ and every $\alpha \neq 0$, we have

$$
\int_{\varepsilon}^{\infty} \frac{e^{i \alpha x}-P_{q-2}(i \alpha x)}{x^{q}} d x=c_{q} \alpha^{q-1}+\frac{(i \alpha)^{q-1}}{(q-1) !} \mathrm{E}_{\varepsilon}(\alpha)+\omega(\varepsilon),
$$

where $c_{q}=\frac{i^{q-1}}{(q-1) !} \sum_{k=0}^{q-2} \frac{1}{k+1}$ for $q \geq 2$ and $c_{1}=0$.

Proof: The proof goes by induction on $q$. If $q=1$, equality holds with $\omega(\varepsilon)=0$. Then, we prove the formula for $q+1$, assuming it true for $q \geq 1$. Since $P_{q-1}^{\prime}=P_{q-2}$, an integration by parts yields 


$$
\int_{\varepsilon}^{\infty} \frac{e^{i \alpha x}-P_{q-1}(i \alpha x)}{x^{q+1}} d x=\frac{e^{i \alpha \varepsilon}-P_{q-1}(i \alpha \varepsilon)}{q \varepsilon^{q}}+\frac{i \alpha}{q} \int_{\varepsilon}^{\infty} \frac{e^{i \alpha x}-P_{q-2}(i \alpha x)}{x^{q}} d x
$$

By the inductive hypothesis,

$$
\frac{i \alpha}{q} \int_{\varepsilon}^{\infty} \frac{e^{i \alpha x}-P_{q-2}(i \alpha x)}{x^{q}} d x=\frac{i c_{q}}{q} \alpha^{q}+\frac{(i \alpha)^{q}}{q !} \mathrm{E}_{\varepsilon}(\alpha)+\omega_{q}(\varepsilon)
$$

for some function $\omega_{q}$ vanishing at 0 . Noting that

$$
\varpi_{q}(\varepsilon)=-\frac{(i \alpha)^{q}}{q ! q}+\frac{e^{i \alpha \varepsilon}-P_{q-1}(i \alpha \varepsilon)}{q \varepsilon^{q}} \rightarrow 0 \quad \text { as } \varepsilon \rightarrow 0,
$$

we end up with the equality

$$
\int_{\varepsilon}^{\infty} \frac{e^{i \alpha x}-P_{q-1}(i \alpha x)}{x^{q+1}} d x=\left[\frac{i^{q}}{q ! q}+\frac{i c_{q}}{q}\right] \alpha^{q}+\frac{(i \alpha)^{q}}{q !} \mathrm{E}_{\varepsilon}(\alpha)+\omega_{q}(\varepsilon)+\varpi_{q}(\varepsilon) .
$$

The final observation that $\frac{i^{q}}{q ! q}+\frac{i c_{q}}{q}=c_{q+1}$ completes the proof.

Proof of Theorem 1 for the case $n+q$ even. Substituting the expression given by Lemma 3 into (2) and noting that

$$
\mathrm{E}_{\varepsilon}(n-2 k)-\mathrm{E}_{\varepsilon}(-(n-2 k))=2 i \operatorname{Si}((n-2 k) \varepsilon),
$$

where

$$
\operatorname{Si}(t)=\int_{t}^{\infty} \frac{\sin x}{x} d x
$$

is the SinIntegral function, we obtain

$$
I_{n, q}=\frac{(-1)^{\frac{q-n}{2}}}{2^{n-1}(q-1) !} \sum_{k=0}^{\left\lfloor\frac{n-1}{2}\right\rfloor}(-1)^{k}\left(\begin{array}{l}
n \\
k
\end{array}\right)(n-2 k)^{q-1} \operatorname{Si}((n-2 k) \varepsilon)+\omega(\varepsilon) .
$$

Since

$$
\mathrm{Si}((n-2 k) \varepsilon) \rightarrow \operatorname{Si}(0)=\frac{\pi}{2} \quad \text { as } \varepsilon \rightarrow 0
$$

the result follows.

Proof of Theorem 1 for the case $n+q$ odd. Again, we substitute the expression given by Lemma 3 into (2). Using (3) and noting that

$$
\mathrm{E}_{\varepsilon}(n-2 k)+\mathrm{E}_{\varepsilon}(-(n-2 k))=2 \mathrm{Ci}((n-2 k) \varepsilon),
$$

where

$$
\mathrm{Ci}(t)=\int_{t}^{\infty} \frac{\cos x}{x} d x
$$


is the CosIntegral function, we obtain

$$
I_{n, q}=\frac{(-1)^{\frac{q-n-1}{2}}}{2^{n-1}(q-1) !} \sum_{k=0}^{\left\lfloor\frac{n-1}{2}\right\rfloor}(-1)^{k}\left(\begin{array}{l}
n \\
k
\end{array}\right)(n-2 k)^{q-1} \operatorname{Ci}((n-2 k) \varepsilon)+\omega(\varepsilon) .
$$

By a further use of (3), we can replace $\mathrm{Ci}((n-2 k) \varepsilon)$ with

$$
\mathrm{Ci}((n-2 k) \varepsilon)-\mathrm{Ci}(\varepsilon) \rightarrow-\log (n-2 k) \quad \text { as } \varepsilon \rightarrow 0,
$$

and a final limit $\varepsilon \rightarrow 0$ completes the argument.

\section{References}

[1] Weisstein ES. Sinc function. Available from: https:/mathworld.wolfram.com/SincFunction.html.

[2] Ahlfors LV. Complex analysis. New York: McGraw-Hill; 1978.

\section{Corresponding author}

Vittorino Pata can be contacted at: vittorino.pata@polimi.it

For instructions on how to order reprints of this article, please visit our website: 